\title{
Estudo de cerâmicas do sítio arqueológico Água Limpa (SP) empregando um equipamento portátil de EDXRF
}

\author{
Fábio L. Melquiades* \\ Carlos Roberto Appoloni* * \\ Taís Pagoto Bélo *** \\ Márcia Angelina Alves ***
}

\begin{abstract}
MELQUIADES, F.L.; APPOLONI, C.R.; BÉLO,_T.P.; ALVES, M.A. Estudo de cerâmicas do sitio arqueológico Água Limpa (SP) empregando um equipamento portátil de EDXRF. Revista do Museu de Arqueologia e Etnologia, São Paulo, 20: 393. 401, 2010.
\end{abstract}

Resumo: $O$ objetivo deste artigo é identificar os elementos químicos presentes em fragmentos cerâmicos do sítio Água Limpa, e associá-los a vários fatores como tecnologia de produção, pigmentação e proveniência. Foi realizada uma análise por Fluorescência de Raios X com Dispersão em Energia (EDXRF) de um conjunto significativo de amostras cerâmicas. A análise é rápida, não destrutiva e sem preparação de amostra. Foram quantificados $\mathrm{Ca}, \mathrm{Ti}, \mathrm{Fe}, \mathrm{Mn}$, $\mathrm{Zn}$, Rb e Zr, possibilitando caracterizar a composição da pasta cerâmica e os elementos chave da pasta empregada nas pigmentações.

Palavras-chave : Fluorescência de Raios X - Cerâmica - Análise não destrutiva.

\section{Introdução}

s ciências físicas têm contribuído sobremaneira nos estudos etnoarqueológicos e arqueológicos sobre a cadeia de eventos pelos quais passam os artefatos cerâmicos desde sua produção, uso, manutenção e armazenagem

(*) Departamento de Fisica, Universidade Estadual do Centro-Oeste.<fmelquiades@unicentro.br>

$\left.{ }^{(* *}\right)$ Departamento de Fisica, Universidade Estadual de Londrina-UEL. <appoloni@uel.br>

${ }^{(* * *)}$ Mestre em arqueologia pelo Museu de Arqueologia e Etnologia da Universidade de São Paulo. 〈taispbelo@gmail.com > ${ }^{(* * *)}$ Museu de Arqueologia e Etnologia da Universidade de São Paulo. <alvesma@usp.br> até chegar ao estágio de seu descarte definitivo. Os dados por elas fornecidos auxiliam na reconstrução da trajetória artefatual, contribuindo para o entendimento do comportamento tecnológico das populações arqueológicas e etnográficas investigadas (Silva e Appoloni 2004)

Especificamente, a cerâmica arqueológica brasileira tem sido usualmente investigada somente através de métodos tradicionais, essencialmente empregando classificações visuais e macroscópicas. Além dos métodos de datação, nenhuma outra técnica nuclear arqueométrica tem sido correntemente utilizada para as investigações de cerâmicas, a não ser em alguns poucos casos recentes (Appoloni 
1997). Foi feita uma breve revisão de diferentes técnicas analiticas empregadas nos últimos anos na análise de cerâmicas para caracterizar sua composição(Akemy et. al. 2007).

Dentre as técnicas analiticas, a Fluorescência de Raios X por Dispersão em Energia (EDXRF) permite a identificação e quantificação, de forma não destrutiva e simultânea, dos diferentes elementos quimicos que constituem uma amostra possibilitando determinar a composição química da pasta cerâmica, bem como dos pigmentos utilizados nos acabamentos de superficie de fragmentos cerâmicos.

O Sitio Água Limpa é um assentamento pré-colonial, localizado no vale da Serra do Jabuticabal, distante $7 \mathrm{~km}$ do municipio de Monte Alto, no norte do estado de São Paulo. A maior questão que envolve a cerâmica do sítio Água Limpa é a compreensão tecnológica de todo processo de fabricação das peças cerâmicas. Por isso, a caracterização dos elementos que compõem a argila, a pintura e o engobo dessa cultura material é fundamental para ser realizada uma comparação com os elementos disponiveis na região, o que pode resultar na compreensão das escolhas do grupo que viveu neste local.

Este trabalho se insere dentro de uma ampla pesquisa cujo objetivo é mapear e pesquisar sítios arqueológicos situados no municipio de Monte Alto, para elaborar a história indigena do vale do Turvo; delinear, a partir da pesquisa empírica de campo, o processo de desenvolvimento cultural do povoamento pré-colonial e de contato centrando na continuidade e mudança cultural (diversidade crono-cultural); estabelecer o modo de vida de populações pré-coloniais associado ao modo de produção; reconstruir o processo produtivo da cerâmica, do lítico e dos artefatos em ossos, das fontes de matérias primas às áreas de caça, pesca e coleta, à elaboração e emprego social do artefato (e sua reutilização) (Bélo 2007).

O estudo tecnológico do processo de produção de cerâmica é essencial. Segundo Leroi-Gourhan (1972), a tecnologia é a ferramenta que permite apreender os primeiros atos propriamente humanos e acompanhá-los de tempo em tempo até o limiar dos tempos atuais através do estudo sistemático de gestos técnicos que indicam continuidade ou mudança cultural. O objetivo deste artigo é identificar os elementos químicos, associados a compostos inorgânicos, presentes em fragmentos cerâmicos do sítio Água Limpa. Para tanto foram realizadas análises nas cerâmicas por EDXRF, visando identificar os elementos químicos presentes em cada fragmento e associar isto a diferentes fatores como tecnologia de produção e proveniência das peças. Este objetivo é primordial para se entender a dinâmica da organização da ocupação desse sítio.

\section{Parte experimental}

\section{Origem das amostras}

O Sitio Água Limpa ( $\left.21^{\circ} 16^{\prime} \mathrm{S}, 48^{\circ} 33^{\prime} \mathrm{W}\right)$ é um sítio a céu aberto, classificando-se no padrão definido por Pallestrini (1975) de sitio 'lito-cerâmico colinar'. É contornado ao sul, pelo córrego Água Limpa e, ao norte, pelo córrego Santa Luzia (Fig. 1).

A geologia do sítio está inserida na Formação Marília, que é sub-grupo do Grupo Bauru. A Formação Marilia consiste de uma sequência de arenitos calciferos, conglomerados e para conglomerados carbonáticos, calcários homogêneos, brechas carbonáticas e lamitos, depositados em sistema de rios entrelaçados, na porção mediana a distal de leques aluviais (Fernandes 1998), sob condições de clima quente seco a levemente úmido (Alves e Ribeiro 1999).

O sitio Água Limpa foi dividido em três zonas de escavação, porém até o momento estão sendo desenvolvidas escavações apenas nas zonas 1 e 2 . A zona 1 corresponde a 1200 $\mathrm{m}^{2}$ e está inserida numa área que antes era pasto para gado e desde 1995 foram plantadas mangueiras no local. A zona 2 corresponde a $5865,60 \mathrm{~m}^{2}$, em cuja área se cultiva cebola e/ou lima da Pérsia.

O método de escavação utilizado foi de "Superficies amplas em decapagens por camadas naturais" adaptado ao solo tropical brasileiro por Pallestrini 1975. O procedimento de 


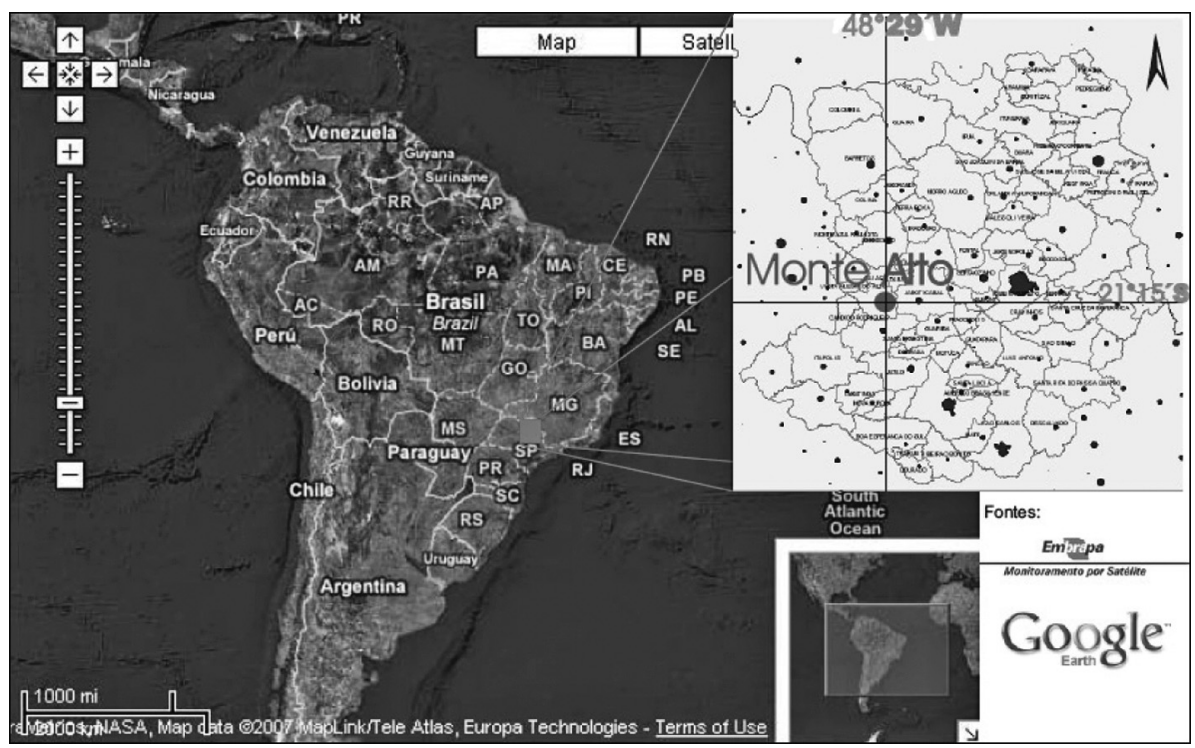

Fig. 1. Mapa da região e do local de amostragem.

campo no sitio consistiu na execução de perfis estratigráficos, trincheiras e decapagens nas zonas 1 e 2 de escavação; perfis, responsáveis pela comprovação de estratos lito-cerâmicos e subquadriculamento em manchas escuras evidenciadas onde foram desenvolvidas decapagens.

Foram analisados 11 fragmentos cerâmicos representativos do material escavado, posicionados diretamente no sistema de medidas, após efetuada uma limpeza cuidadosa das superfícies externas. Sendo assim, a amostra foi analisada in natura. As amostras foram medidas dos dois lados e transversalmente, sendo considerados dois ou três pontos diferentes de cada lado da amostra, dependendo do tamanho da peça, e dois pontos transversalmente. Sendo assim, em cada amostra foram realizadas pelo menos seis medidas.

\section{Critérios para análise dos} fragmentos cerâmicos

Os critérios analíticos para análise tecnotipológica levam em consideração a argila, cerâmica, formas (base, bojo e borda), pintura, decoração plástica, queima, tempero/antiplástico, pasta, resistência mecânica da cerâmica, minerais e outros.
A técnica utilizada para elaboração dos vasilhames cerâmicos é a acordelada (os artefatos são construídos da base em direção ao corpo, bordas e lábios através da execução e distribuição circular de roletes de argila). Aparecem como acabamento de superficie o alisamento e o polimento, e como decoração plástica o uso de pintura vermelha, engobo branco, banho vermelho e brunidura. A queima foi provavelmente através de fogueira rasa, próxima e externa às habitações.

\section{Metodologia de análise e quantificação}

A técnica de Fluorescência de raios X é tradicionalmente utilizada para quantificação de elementos inorgânicos (Injuk e Van Grieken 2002; Jenkins 1999). Mesmo usando equipamentos portáteis ou de bancada, diversos aspectos atrativos e vantagens podem ser combinados: simplicidade, medidas rápidas, metodologia não destrutiva, identificação e/ou quantificação simultânea e a possibilidade de realizar medidas in situ. A teoria da Fluorescência de Raios X está baseada no efeito fotoelétrico, isto é, elétrons localizados nas camadas mais internas dos átomos são removidos. Promover estas transições exige uma fonte com energia suficiente, tais como fontes radioativas, tubos 
de raios X, particulas aceleradas ou radiação sincrotron. Elétrons das camadas mais externas ocupam a vacância eletrônica resultante da excitação, produzindo a emissão de raios $\mathrm{X}$ caracteristicos. Estas emissões têm energias equivalentes às diferenças entre as energias potenciais dos orbitais envolvidos nas transições (Alves e Ribeiro 1999).

A metodologia de quantificação das amostras consistiu na medida de quatro padrões certificados de solos e argilas. Os padrões medidos foram IAEA-Soil7, SARM69, Argila Plástica IPT-padrão 32 e Argila Pará IPTpadrão 28 , todos eles na forma de pastilhas. Com os resultados de intensidade dos padrões obtiveram-se as curvas de concentração para cada elemento.

\section{Equipamento de medida por EDXRF}

As medidas de EDXRF foram realizadas no Laboratório de Física Nuclear Aplicada da Universidade Estadual de Londrina (LFNAUEL) utilizando o sistema PXRF-LFNA-02 composto por: um detector de raios $\mathrm{X}$ tipo $\mathrm{Si}$ PIN (FWHM de $221 \mathrm{eV}$ para a linha de 5,9 $\mathrm{keV}$, janela de Be de $25 \mu \mathrm{m}$ ) e um mini tubo de raios $\mathrm{X}$ (alvo de $\mathrm{Ag}$, 4W, filtro de $\mathrm{Ag}$ com 50 $\mu \mathrm{m}$, foco de aproximadamente $10 \mathrm{~mm}$ de diâmetro). Cada irradiação foi realizada com $28 \mathrm{kV}$ e $5 \mu \mathrm{A}$, durante 500 s.

\section{Resultados e discussão}

Nas amostras foram quantificados Ca, Ti e $\mathrm{Fe}$, como elementos majoritários e $\mathrm{Mn}, \mathrm{Zn}, \mathrm{Rb}$ e $\mathrm{Zr}$ como elementos minoritátios, com concentrações que não ultrapassam a ordem de dezenas de $\mathrm{mg} \mathrm{Kg}^{1}$. A Tabela 1 apresenta as equações de calibração obtidas a partir dos padrões multielementares. Os resultados das concentrações obtidas estão na Tabela 2.

As amostras 1, 2 e 3 são do mesmo local de coleta, porém de profundidades diferentes. As amostras 9, 10 e 11 apresentam cor avermelhada em sua totalidade ou em pontos do fragmento. A Tabela 3 apresenta comentários interpretando os resultados obtidos.
Tabela 1

\begin{tabular}{|c|c|c|}
\hline \multicolumn{3}{|c|}{$\begin{array}{c}\text { Equações de calibração com seus respectivos } \\
\text { valores do coeficiente de determinação }\end{array}$} \\
\hline Elemento & Equação de calibração & $\mathrm{R} 2$ \\
\hline $\mathrm{Ca}$ & $\mathrm{C}=0,0401 \mathrm{I}$ & 0,925 \\
\hline $\mathrm{Ti}$ & $C=0,0525 \mathrm{I}+0,1015$ & 0,982 \\
\hline $\mathrm{Mn}$ & $C=0,0021 \mathrm{I}+0,0442$ & 0,998 \\
\hline $\mathrm{Fe}$ & $C=0,0095 I+0,1831$ & 0,974 \\
\hline $\mathrm{Zn}$ & $C=0,6538 \mathrm{I}$ & 0,998 \\
\hline $\mathrm{Rb}$ & $C=1,2952 \mathrm{I}$ & 0,998 \\
\hline $\mathrm{Zr}$ & $C=1,4694 \mathrm{I}$ & 0,998 \\
\hline
\end{tabular}

$\mathrm{C}=$ concentração do elemento

$\mathrm{I}=$ intensidade liquida medida

As amostras 1, 2, 3, 7 e 10 apresentam concentração dos elementos medidos em um dos lados maior que do outro e maior que a pasta cerâmica, indicando a presença de engobo. As amostras 4,5 e 8 não apresentaram diferenças significativas entre o lado interno, externo e transversal, podendo ser consideradas como peças homogêneas e sem nenhum tipo de acabamento. As amostras 6 e 11 foram encontradas no mesmo local, confirmando o dominio do processo de coloração da cerâmica e do acabamento com engobo. As peças 9 e 10 apresentaram maior concentração de ferro nos lados avermelhados e supõe-se o uso de matéria prima com óxido de ferro para obtenção de tal coloração.

A amostra 10, apresentada na Fig. 2 é uma peça com detalhes refinados de acabamento. Existe uma mancha vermelha com concentração de ferro mais elevada e um fundo branco onde a presença de titânio é acentuada. Os espectros para cada um dos lados desta amostra estão na Fig. 3.

\section{Análise de clusters}

A Fig. 4 apresenta o resultado da análise multivariada pelo método de Ward, usando as concentrações dos elementos Ti, Fe e Zr.

A análise do dendrograma indica três diferentes fontes de argila. No entanto, dado o pequeno número de fragmentos, esta indicação deve ser tomada com muito cuidado, em 
Tabela 2

\begin{tabular}{lccccccc}
\hline \multicolumn{5}{c}{ Valores de concentração para cada fragmento cerâmico analisado } \\
\hline \hline \multicolumn{5}{c}{ Amostra 1 } & \multicolumn{2}{c}{ Amostra 2 } & \multicolumn{2}{c}{ Amostra 3 } \\
\hline Elemento (unidade) & Lado A & Lado B & Lado C & Lado A & Lado B & Lado A & Lado B \\
\hline $\mathrm{Ca}(\%)$ & & & & & & $0,24 \pm 0,07$ & $0,99 \pm 0,27$ \\
$\mathrm{Ti}(\%)$ & $0,78 \pm 0,05$ & $1,36 \pm 0,05$ & $0,84 \pm 0,03$ & $1,30 \pm 0,04$ & $1,68 \pm 0,04$ & $3,00 \pm 0,08$ & $4,91 \pm 0,10$ \\
$\mathrm{Fe}(\%)$ & $4,32 \pm 0,03$ & $7,75 \pm 0,04$ & $5,30 \pm 0,01$ & $2,78 \pm 0,02$ & $3,77 \pm 0,02$ & $2,71 \pm 0,09$ & $3,77 \pm 0,10$ \\
$\mathrm{Mn}(\mathrm{mg} / \mathrm{Kg})$ & & & & & $0,07 \pm 0,01$ & $0,08 \pm 0,01$ \\
$\mathrm{Zn}(\mathrm{mg} / \mathrm{Kg})$ & $3,37 \pm 0,40$ & $6,09 \pm 0,39$ & $0,52 \pm 0,20$ & & & $1,51 \pm 0,43$ & $2,50 \pm 0,53$ \\
$\mathrm{Rb}(\mathrm{mg} / \mathrm{Kg})$ & $3,80 \pm 1,50$ & $6,70 \pm 1,30$ & $5,80 \pm 0,70$ & $5,80 \pm 1,00$ & $8,10 \pm 1,10$ & & \\
$\mathrm{Zr}(\mathrm{mg} / \mathrm{Kg})$ & $13,9 \pm 5,00$ & $17,80 \pm 4,90$ & $15,30 \pm 2,20$ & $23,90 \pm 4,00$ & $32,00 \pm 4,50$ & $34,20 \pm 6,80$ & $38,10 \pm 8,30$ \\
\hline
\end{tabular}

Tabela 2 (cont.)

Valores de concentração para cada fragmento cerâmico analisado

\begin{tabular}{lcrrrrrr}
\hline \hline & \multicolumn{2}{c}{ Amostra 4 } & \multicolumn{2}{c}{ Amostra 5 } & \multicolumn{3}{c}{ Amostra 6 } \\
\hline Elemento (unidade) & Lado A & Lado B & Lado A & Lado B & Lado A & Lado B & Lado C \\
\hline $\mathrm{Ca}(\%)$ & & & & & $0,18 \pm 0,02$ & $0,06 \pm 0,03$ & $0,07 \pm 0,02$ \\
$\mathrm{Ti}(\%)$ & $1,26 \pm 0,06$ & $1,75 \pm 0,05$ & $1,24 \pm 0,05$ & $1,59 \pm 0,06$ & $0,39 \pm 0,04$ & $0,50 \pm 0,05$ & $0,19 \pm 0,05$ \\
$\mathrm{Fe}(\%)$ & $3,79 \pm 0,03$ & $3,76 \pm 0,02$ & $2,14 \pm 0,08$ & $2,23 \pm 0,09$ & $3,17 \pm 0,07$ & $5,33 \pm 0,11$ & $2,02 \pm 0,06$ \\
$\mathrm{Mn}(\mathrm{mg} / \mathrm{Kg})$ & & & $0,06 \pm 0,01$ & $0,06 \pm 0,01$ & $0,07 \pm 0,01$ & $0,09 \pm 0,01$ & $0,06 \pm 0,01$ \\
$\mathrm{Zn}(\mathrm{mg} / \mathrm{Kg})$ & $4,22 \pm 0,25$ & $5,21 \pm 0,39$ & $2,25 \pm 0,40$ & $2,15 \pm 0,45$ & & $1,22 \pm 0,28$ & \\
$\mathrm{Rb}(\mathrm{mg} / \mathrm{Kg})$ & $7,5 \pm 0,8$ & $8,60 \pm 1,10$ & $5,40 \pm 1,20$ & $6,20 \pm 1,30$ & & & \\
$\mathrm{Zr}(\mathrm{mg} / \mathrm{Kg})$ & $44,4 \pm 3,7$ & $38,10 \pm 5,50$ & $26,20 \pm 4,90$ & $31,20 \pm 5,10$ & $9,70 \pm 1,10$ & $8,00 \pm 3,10$ & $27,00 \pm 10,80$ \\
\hline
\end{tabular}

Tabela 2 (cont.)

\begin{tabular}{lrrrrrr}
\hline \multicolumn{5}{c}{ Valores de concentração para cada fragmento cerâmico analisado } \\
\hline \hline \multicolumn{5}{c}{ Amostra 7} & \multicolumn{3}{c}{ Amostra 8} \\
\hline Elemento (unidade) & \multicolumn{1}{c}{ Lado A } & \multicolumn{1}{c}{ Lado B } & \multicolumn{1}{c}{ Lado C } & Lado A & Lado B & Lado C \\
\hline $\mathrm{Ca}(\%)$ & $0,44 \pm 0,06$ & $0,43 \pm 0,06$ & $0,08 \pm 0,04$ & & & \\
$\mathrm{Ti}(\%)$ & $1,67 \pm 0,04$ & $1,46 \pm 0,05$ & $0,87 \pm 0,04$ & $1,36 \pm 0,06$ & $1,49 \pm 0,06$ & $1,38 \pm 0,04$ \\
$\mathrm{Fe}(\%)$ & $5,63 \pm 0,03$ & $5,82 \pm 0,07$ & $4,40 \pm 0,06$ & $2,44 \pm 0,10$ & $3,35 \pm 0,11$ & $3,09 \pm 0,06$ \\
$\mathrm{Mn}(\mathrm{mg} / \mathrm{Kg})$ & & & $0,08 \pm 0,01$ & $0,06 \pm 0,01$ & $0,08 \pm 0,01$ & $0,07 \pm 0,01$ \\
$\mathrm{Zn}(\mathrm{mg} / \mathrm{Kg})$ & $3,49 \pm 0,28$ & $4,06 \pm 0,33$ & $3,63 \pm 0,25$ & $2,24 \pm 0,46$ & $3,12 \pm 0,50$ & $3,48 \pm 0,28$ \\
$\mathrm{Rb}(\mathrm{mg} / \mathrm{Kg})$ & $6,70 \pm 1,00$ & $6,30 \pm 1,00$ & $5,40 \pm 0,70$ & $6,50 \pm 1,50$ & $7,80 \pm 1,50$ & $5,10 \pm 0,80$ \\
$\mathrm{Zr}(\mathrm{mg} / \mathrm{Kg})$ & $24,60 \pm 4,20$ & $16,40 \pm 4,40$ & $21,10 \pm 2,90$ & $30,30 \pm 5,60$ & $36,90 \pm 5,60$ & $31,80 \pm 3,40$ \\
\hline
\end{tabular}

Tabela 2 (cont.)

Valores de concentração para cada fragmento cerâmico analisado

\begin{tabular}{lcccccrr}
\hline \hline & \multicolumn{2}{c}{ Amostra 9 } & \multicolumn{3}{c}{ Amostra 10 } & \multicolumn{2}{c}{ Amostra 11 } \\
\hline Elemento (unidade) & Lado A & Lado B & Lado A & Lado B & Lado C & Lado A & Lado B \\
\hline $\mathrm{Ca}(\%)$ & & & & & & & \\
$\mathrm{Ti}(\%)$ & $2,36 \pm 0,07$ & $3,51 \pm 0,06$ & $3,51 \pm 0,06$ & $1,49 \pm 0,03$ & $0,83 \pm 0,02$ & $1,37 \pm 0,07$ & $1,26 \pm 0,08$ \\
$\mathrm{Fe}(\%)$ & $6,43 \pm 0,02$ & $3,09 \pm 0,02$ & $3,09 \pm 0,02$ & $1,93 \pm 0,06$ & $1,60 \pm 0,04$ & $11,59 \pm 0,18$ & $10,08 \pm 0,17$ \\
$\mathrm{Mn}(\mathrm{mg} / \mathrm{Kg})$ & & & & $0,05 \pm 0,01$ & $0,05 \pm 0,01$ & $0,10 \pm 0,01$ & $0,11 \pm 0.01$ \\
$\mathrm{Zn}(\mathrm{mg} / \mathrm{Kg})$ & & & & $3,34 \pm 0,27$ & $4,80 \pm 0,21$ & $4,25 \pm 0.54$ & $4,08 \pm 0.58$ \\
$\mathrm{Rb}(\mathrm{mg} / \mathrm{Kg})$ & & & & & & $6,80 \pm 1,70$ & $8,70 \pm 1,90$ \\
$\mathrm{Zr}(\mathrm{mg} / \mathrm{Kg})$ & $41,90 \pm 3,20$ & $54,90 \pm 4,80$ & $54,90 \pm 4,80$ & $20,50 \pm 3,00$ & $23,20 \pm 2,20$ & $25,60 \pm 5,70$ & $25,60 \pm 6,00$ \\
\hline
\end{tabular}


Tabela 3

\begin{tabular}{|c|c|c|}
\hline \multicolumn{3}{|c|}{ Interpretação dos resultados de concentração para cada amostra } \\
\hline $\mathrm{N}^{\circ}$ & Amostra & Resultados da análise por EDXRF \\
\hline 1 & $\begin{array}{l}\text { Amostra AL95m8 } \\
\text { Z2P1, } \\
\text { m: } 8 \text { a } 10 \\
\text { profundidade } 46 \mathrm{~cm}\end{array}$ & $\begin{array}{l}\text { Maior quantidade de Fe e Ti do lado B, indicando a presença } \\
\text { de engobo. }\end{array}$ \\
\hline 2 & $\begin{array}{l}\text { Amostra AL95m } 15 \\
\text { Z2P1, } \\
\text { m: } 15 \text { a } 18, \\
\text { profundidade } 51 \text { a } 92 \mathrm{~cm}, \\
\text { entre T4 e T5 }\end{array}$ & $\begin{array}{l}\text { No lado externo a concentração dos elementos é maior } \\
\text { indicando a presença de engobo. }\end{array}$ \\
\hline 3 & $\begin{array}{l}\text { Amostra AL95m0 } \\
\text { AL95 Z2P1, } \\
\mathrm{m}: 0-5, \\
\text { profundidade } 58 \text { A } 77 \mathrm{~cm}\end{array}$ & $\begin{array}{l}\text { O lado externo apresenta maior concentração dos elementos } \\
\text { encontrados indicando a presença de engobo. }\end{array}$ \\
\hline 4 & $\begin{array}{l}\text { Amostra AL96 } \\
\text { Z2P1T5, } \\
\text { profundidade } 50-70 \mathrm{~cm}\end{array}$ & $\begin{array}{l}\text { Neste caso os dois lados da amostra apresentam concentrações } \\
\text { semelhantes e maiores que da pasta, indicando a presença de } \\
\text { engobo nos dois lados. }\end{array}$ \\
\hline 5 & $\begin{array}{l}\text { Amostra AL96m8 } \\
\text { Z2S1M8P1, } \\
\text { profundidade } 50-100 \mathrm{~cm} \text { parede sul } \\
12 / 09\end{array}$ & Os dois lados apresentam concentrações estatisticamente iguais. \\
\hline 6 & $\begin{array}{l}\text { Amostra AL9544 } \\
\text { AL95 Z2P1, } \\
\text { m: } 0 \text { - 5, profundidade } 58 \text { A } 77 \mathrm{~cm}\end{array}$ & $\begin{array}{l}\text { Esta peça foi trabalhada interna e externamente. Isto pode ser } \\
\text { verificado com a maior concentração das partes externas em } \\
\text { relação à pasta cerâmica medida no lado C. }\end{array}$ \\
\hline 7 & $\begin{array}{l}\text { Amostra ALO0 } \\
\text { S2T2, } \\
\text { Coleta na peneira, } \\
16 / 08 / 2000\end{array}$ & $\begin{array}{l}\text { Percebe-se que as concentrações de Ca, Ti e Fe são maiores na } \\
\text { parte externa do que na pasta cerâmica, indicando a presença } \\
\text { de engobo dos dois lados. }\end{array}$ \\
\hline \multirow[t]{2}{*}{8} & $\begin{array}{l}\text { AL } 98 \text { Z2S1M8P1 } \\
\text { profundidade } 50-100 \mathrm{~cm} \text { parede sul } \\
12 / 09 \text { - AL98 } \mathrm{m} 8\end{array}$ & $\begin{array}{l}\text { O lado B e C apresentam maiores concentrações de ferro do que } \\
\text { o lado A. Contudo, devido a pequena diferença nas concentrações } \\
\text { verifica-se uma semelhança na composição geral do artefato. }\end{array}$ \\
\hline & Fragmentos vermelhos & \\
\hline 9 & $\begin{array}{l}\text { Amostra AL96m1 } \\
\text { Z2P1T5, } \\
\text { m: } 1 \text {, } \\
\text { profundidade } 70-80 \mathrm{~cm}, \\
\text { 16/set }\end{array}$ & $\begin{array}{l}\text { A parte interna (lado A) apresenta maior quantidade de Fe, cuja } \\
\text { presença como elemento chave justifica a tonalidade avermelhada, } \\
\text { quando comparada com o lado externo. }\end{array}$ \\
\hline 10 & $\begin{array}{l}\text { Amostra AL96Z1 } \\
\text { Z1, coleta de superfície, } \\
\text { 03/09/96 }\end{array}$ & $\begin{array}{l}\text { A presença de engobo foi comprovada, visto que a medida de } \\
\text { cada um dos lados apresenta maior concentração de Fe e Ti do } \\
\text { que a pasta cerâmica, sendo que o lado A é mais rico em Fe e } \\
\text { Ti. Comparando a medida do ponto } 1 \text { (sobre a pintura verme- } \\
\text { lha) com a do ponto } 5 \text { (sobre o engobo branco), verificamos que } \\
\text { a quantidade de ferro sobre o ponto } 1 \text { é maior que a no ponto } \\
5 \text {, indicando que o ferro é o elemento chave da matéria prima } \\
\text { utilizada na tinta vermelha. Além disso, a quantidade de Ti é } \\
\text { menor no ponto } 1 \text { do que no ponto } 5 \text {, indicando que o } \\
\text { elemento chave da matéria prima do engobo branco é o Ti. }\end{array}$ \\
\hline 11 & $\begin{array}{l}\text { Amostra AL95m } 10 \\
\text { AL95 Z2P1, } \\
\text { m: } 0-5, \\
\text { profundidade } 58 \text { A } 77 \mathrm{~cm}\end{array}$ & $\begin{array}{l}\text { Nesta peça a concentração de Fe é muito alta, em comparação } \\
\text { com as demais peças, o que indica que a coloração avermelhada } \\
\text { é devido à presença de alta concentração de óxido de ferro na } \\
\text { matéria prima utilizada. }\end{array}$ \\
\hline
\end{tabular}


Fábio L. Melquiades Carlos Roberto Appoloni Tais Pagoto Bélo

Márcia Angelina Alves
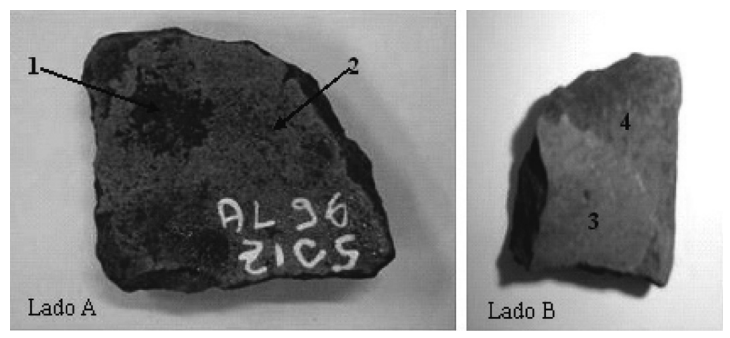

Fig. 2. Amostra 10, lado A e lado B. Os números indicam os locais irradiados para análise por EDXRF.

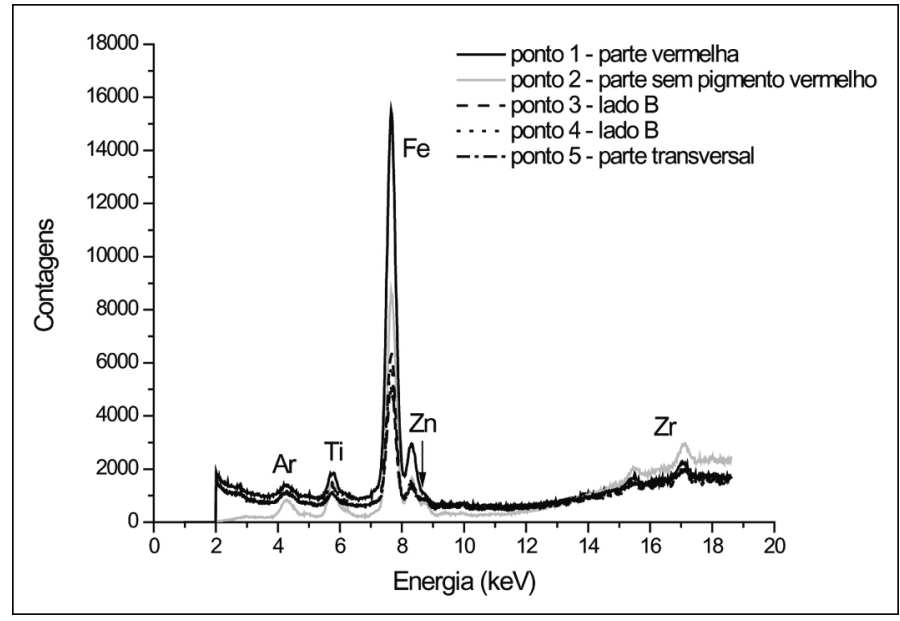

Fig. 3. Espectros da amostra 10, mostrando os 5 pontos irradiados em 3 lados da amostra.

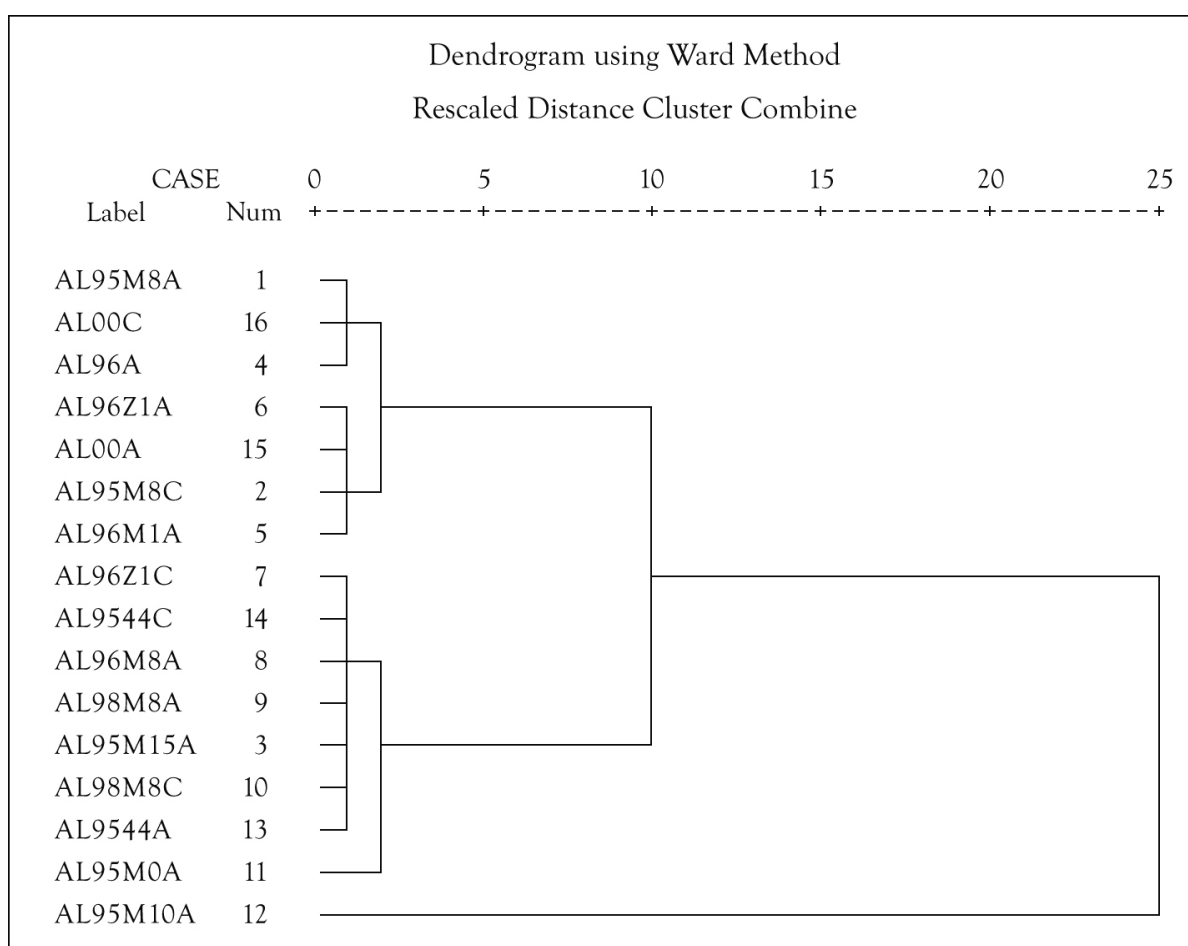

Fig. 4. Dendrograma da análise pelo método de Ward para as argilas dos fragmentos analisados. 
especial para a amostra 10 lado A, que ficou separada de todas as outras, devido à alta concentração de ferro na argila deste fragmento.

\section{Conclusões}

A argila utilizada para a fabricação da cerâmica era possivelmente obtida de fontes argilosas próximas ao sítio, provavelmente nos córregos Santa Luzia e Água Limpa. Este fato certifica-se pela análise por EDXRF, visto que a concentração de ferro $(1,60$ a 5,30\%) e titânio $(0,19$ a $1,38 \%)$ encontrada nas amostras está de acordo com a quantidade de ferro $(1,29$ a $2,60 \%)$ e titânio $(0,27$ a $1,05 \%)$, determinadas por Gravina et al. (2002), que compõe o solo da Formação Marilia, onde se encontra o sítio.

Em todas as amostras foram encontrados Fe e Ti, provavelmente devido à composição do solo por latossolo roxo e vermelho escuro. Contudo, em grande parte das amostras a concentração de Fe e Ti foi maior do lado externo das peças, indicando a presença de engobo. As amostras com pintura vermelha e banho vermelho apresentaram maior quantidade de Fe no local da superfície em que se encontravam presentes os pigmentos, indicando claramente ser este o elemento chave do pigmento. Uma das amostras apresentou engobo branco, e nesta a concentração de Ti foi maior que as demais indicando que é o elemento chave do material utilizado para dar a coloração branca à peça, mas não necessariamente que o pigmento seja um composto de $\mathrm{Ti}$, pois se sabe que o Ti aparece com frequência em caolins, os quais são bastante utilizados como pigmentos brancos em cerâmicas indigenas brasileiras. Foi identificada ainda, em algumas amostras, a presença de $\mathrm{Ca}, \mathrm{Mn}, \mathrm{Zn}$ e Zr.

Através da técnica de EDXRF, com equipamento portátil, foi possivel caracterizar a composição da pasta cerâmica e os elementos chave da pasta empregada nas pigmentações. Além disso, toda análise foi feita de modo não destrutivo e dispensando a preparação de amostra.

MELQUIADES, F.L.; APPOLONI, C.R.; BÉLO, T.P.; ALVES, M.A. Study of ceramic fragments from Água Limpa (SP) archeological site using a portable EDXRF system. Revista do Museu de Arqueologia e Etnologia, São Paulo, 20: 393-401, 2010.

Abstract: The objective of this paper is to identify the chemical elements present in each fragment and associate it to various factors such as production technology, pigmentation and provenance. Energy Dispersive X-ray Fluorescence (EDXRF) analysis of a representative ensemble of ceramic fragments were performed. The analysis is fast, non-destructive and without sample preparation. Calcium, Ti, Fe, Mn, Zn, Rb and Zr were quantified. It was possible to characterize the ceramic paste composition and the key elements of the raw materials employed for the pigmentation.

Keywords: X-ray Fluorescence - Ceramic - Non-destructive analysis. 
Fábio L. Melquiades

\section{Referências bibliográficas}

AKEMY, T. B.; SARKIS, J.E.S.; SALVADOR, V.L.R.;

2007 Análise Arqueométrica de Cerâmica Tupiguarani da Região Central do Estado do Rio Grande do Sul, Brasil, Usando Fluorescência de Raios X por Dispersão de Energia (Edxrf) Quim. Nova, 30: 785-790.

ALVES J.M.P.; RIBEIRO D.T.P.

1999 50 Simpósio sobre o Cretáceo do Brasil, Serra Negra, Brasil.

APPOLONI, C.R.

1997 Estudo de cerâmica arqueológica do Paraná por técnicas Nucleares não destrutivas. Revista do Museu de Arqueologia e Etnologia (USP) 1997, Suplemento 2, 135-149.

BÉLO T.P.

2007 Estudo tecnológico e das cadeias operatórias da cultura material lítica e cerâmica, coletada nos anos de 1995, 1996 e 2000, no sítio Água Limpa, municipio de Monte Alto, SP. Dissertação de mestrado, USP.

FERNANDES L.A.

1998 Contexto paleoambiental de ocorrência de ossos de vertebrados do Grupo Bauru (Ks), Estado do Paraná. Tese de Doutorado, Instituto de Geociências, Universidade de São Paulo.
GRAVINA, E.G.; KAFINO, C.V.; BROD, J.A.; BOAVENTURA, G.R.; SANTOS, R.V.; JOST, H.

2002 Proveniência de Arenitos das Formações Uberaba e Marilia (Grupo Bauru) e do Garimpo do Bandeira: Implicações para a Controvérsia sobre a Fonte do Diamante do Triângulo Mineiro, Revista Brasileira de Geociências, 32: 545-558.

INJUK, J.; VAN GRIEKEN R.

2002 Recent Technological Advances, Wiley, Chichester, 2004, pp. 569-592

JENKIS, R.

1999 X-ray fluorescence spectrometry. New York: Wiley-Interscience.

LEROI GOURHAN, A.;

1972 Vocaubulaire - fouilles de Pincevent: essai d'analyse ethnographique d'un habitat magdalenien. La section 36, Paris: CNRS.

PALLESTRINI, L.

1975 Interpretação das estruturas arqueológicas em sítios do Estado de São Paulo. São Paulo. Série Arqueologia 1. Coleção Museu Paulista, fundo de pesquisa, USP.

SILVA, F.A.; APPOLONI C.R.

2004 A Arqueometria e a Análise de Artefatos Cerâmicos: um estudo de fragmentos cerâmicos etnográficos e arqueológicos por Fluorescência de Raios X (EDXRF) e Transmissão Gama. Revista de Arqueologia 17: 41-61. 